\title{
Non-tilted Bianchi VII 0 models - the radiation fluid
}

\author{
U S Nilsson, M J Hancock, and J Wainwright \\ Department of Applied Mathematics \\ University of Waterloo \\ Waterloo, Ontario \\ Canada, N2L 3G1
}

June 21, 2021

\begin{abstract}
We consider the late time behaviour of non-tilted perfect fluid Bianchi $\mathrm{VII}_{0}$ models when the source is a radiation fluid, thereby completing the analysis of the Bianchi $\mathrm{VII}_{0}$ models initiated by Wainwright et al in a recent paper. The models exhibit the phenomena of asymptotic self-similarity breaking and Weyl-curvature dominance at late times. The late time dynamics of the $\mathrm{VII}_{0}$ perfect fluid models, and in particular that of the radiation fluid, is a prime example of the complexity inherent in the field equations of general relativity.
\end{abstract}

\section{Introduction}

The late time behaviour of Bianchi type $\mathrm{VII}_{0}$ models with a non-tilted perfect fluid source has been shown to exhibit two new dynamic features at late times, namely, the breaking of asymptotic self-similarity and Weyl curvature dominance (see Wainwright et al (1999)円, pages 2587-8). These models therefore serve as counterexamples to the notion that any Bianchi universe is approximated by a self-similar Bianchi universe at late times. The breaking of asymptotic self-similarity is characterized by oscillations in the dimensionless shear scalar that become increasingly rapid in terms of the cosmological clock time $t$ as $t \rightarrow \infty$. This behaviour also leads to the Weyl curvature dominance, which refers to the fact that certain expansionnormalized scalars formed from the Weyl tensor become unbounded as $\tau \rightarrow+\infty$.

It is customary in cosmology to describe the source of the gravitational field in terms of a perfect fluid, for which the energy-momentum tensor has the form

$$
T_{a b}=\mu u_{a} u_{b}+p\left(g_{a b}+u_{a} u_{b}\right)
$$

where $\mu \geq 0$ is the fluid energy density, $p \geq 0$ the fluid pressure, and $u^{a}$ the fluid 4 -velocity. In WHU, the linear equation of state

$$
p=(\gamma-1) \mu,
$$

where the constant $\gamma$ satisfies $\frac{2}{3}<\gamma<2$, was studied. The two cases of primary interest in cosmology are dust $(\gamma=1)$, describing the matter-dominated epoch of the universe, and radiation $\left(\gamma=\frac{4}{3}\right)$, describing the radiation-dominated epoch of the early universe. The late-time behaviour? of a radiation model would describe the final stages of this epoch, prior to the time at which matter and radiation decouple. In WHU it was shown that for Bianchi $\mathrm{VII}_{0}$ models a physically important bifurcation occurs at the value $\gamma=\frac{4}{3}$, namely that the dimensionless shear scalar $\Sigma$ (see Section 4) tends to zero at late times if and only if $\gamma \leq \frac{4}{3}$.

\footnotetext{
${ }^{1}$ From now on we will refer to this paper as WHU

${ }^{2}$ From a dynamical systems point of view, the late time behaviour signifies that the evolution is governed by the future attractor of the dynamical system.
} 
In that paper a complete proof of the late time behaviour of dust models (in fact all models with $\frac{2}{3}<\gamma<\frac{4}{3}$ ) was given, but the results for the radiation models were stated without proof. The goal of the present paper, which should be read in conjunction with WHU, is to remedy this deficiency.

The late time behaviour of the Bianchi $\mathrm{VII}_{0}$ radiation models was first discussed by Doroshkevich et al (1973) (see equation (9) and equations (I.14) and (I.15) in Appendix I), and subsequently interpreted in a series of papers by Lukash $(1974,1975,1976)$. Doroshkevich et al gave the asymptotic forms of the metric coefficients, but did not give a proof or any justification of their results. Reference is made to two preprints that we have been unable to locate (their references 19 and 20). Our approach, which makes use of the orthonormal frame formalism and expansion-normalized variables, provides a convenient framework for proving the results, and is also well suited for numerical simulations. In addition, it has the advantage that it leads directly to the asymptotic behaviour of the dimensionless anisotropy scalars (see Section 4) that describe the deviation of the models from a Friedmann-Lemaitre (FL) model.

The outline of the paper is as follows. In Section 2 we present the evolution equations for Bianchi $\mathrm{VII}_{0}$ models with a radiation fluid. In Section 3 we establish the limits of the dimensionless gravitational field variables at late times. Section 4 is devoted to the question of isotropization and whether the Bianchi $\mathrm{VII}_{0}$ models are close to any FL model at late times. In Section 国 the detailed asymptotic forms of the gravitational field variables are found using centre manifold theory. In Section 6 , the results of the previous Sections are used to determine the asymptotic forms of the metric components, and a comparison with the results of Doroshkevich et al (1973) is made. We conclude in Section 7 with a discussion of the implications of the results. The details of the proofs are given in three appendices.

\section{Evolution equations}

Since the evolution equations for Bianchi $\mathrm{VII}_{0}$ models with a radiation-fluid source can be found by specializing the equations given in WHU, we will only briefly discuss the choice of variables. We use the orthonormal frame approach of Ellis \& MacCallum (1969). The basic variables are $\left\{H, \sigma_{ \pm}, n_{ \pm}\right\}$, where $H$ is the Hubble parameter. The variables $\sigma_{ \pm}$, which parametrize the non-zero components of the shear tensor, describe the anisotropy of the expansion of the cosmological model, while the $n_{ \pm}$describe the spatial curvature of the orbits of the Bianchi $\mathrm{VII}_{0}$ symmetry group. The next step is to introduce expansion-normalized variables according to

$$
\left\{\Sigma_{ \pm}, N_{ \pm}\right\}=\frac{1}{H}\left\{\sigma_{ \pm}, n_{ \pm}\right\}, \quad \Omega=\frac{\mu}{3 H^{2}},
$$

(see Wainwright \& Ellis (1997), page 112, for the motivation of this normalization). The density parameter $\Omega$ is given by

$$
\Omega=1-\Sigma_{+}^{2}-\Sigma_{-}^{2}-N_{-}^{2},
$$

(see equation (3.9) in WHU). The assumption of a non-negative fluid energy density yields $\Omega \geq 0$. This, in conjunction with (国), implies that the variables $\Sigma_{+}, \Sigma_{-}$, and $N_{-}$are bounded. The remaining gravitational field variable, $N_{+}$, however, need not be. In fact, it has been shown in WHU that

$$
\lim _{\tau \rightarrow \infty} N_{+}=\infty,
$$

for all initial states with $\Omega>0$ and $\frac{2}{3}<\gamma<2$. The time-variable $\tau$ is the so-called Hubble-time, which satisfies

$$
\frac{d t}{d \tau}=H^{-1}
$$

where $t$ is the cosmological clock time. The behaviour of $N_{+}$at late times motivates the introduction of a new set of variables $\{R, \psi, M\}$ according to

$$
\Sigma_{-}=R \cos \psi, \quad N_{-}=R \sin \psi, \quad M=\frac{1}{N_{+}},
$$


where $R \geq 0$. The case $R=0$, which results in $\Sigma_{-}=N_{-}=0$, describes the Bianchi VII $_{0}$ locally rotationally symmetric (LRS) models. For these models, $\psi$ is irrelevant, as can be seen from (7). Since Bianchi VII LRS models are equivalent to Bianchi type I LRS models, they are asymptotically self-similar and do not exhibit Weyl curvature dominance at late times. We will therefore not consider this particular class of models further. The evolution equations of the gravitational field are

$$
\begin{aligned}
\Sigma_{+}^{\prime} & =-R^{2}-\Sigma_{+}\left(1-\Sigma_{+}^{2}\right)+\left(1+\Sigma_{+}\right) R^{2} \cos 2 \psi, \\
R^{\prime} & =\left[\left(1+\Sigma_{+}\right) \Sigma_{+}+\left(R^{2}-1-\Sigma_{+}\right) \cos 2 \psi\right] R, \\
M^{\prime} & =-\left[\left(1+\Sigma_{+}\right)^{2}+R^{2}(\cos 2 \psi+3 M \sin 2 \psi)\right] M, \\
\psi^{\prime} & =\frac{1}{M}\left[2+\left(1+\Sigma_{+}\right) M \sin 2 \psi\right],
\end{aligned}
$$

where a prime denotes differentiation with respect to $\tau$. These equations correspond to equations (3.17)(3.22) in WHU with the specific value $\gamma=\frac{4}{3}$. Equation (4) now takes the form

$$
\Omega=1-\Sigma_{+}^{2}-R^{2}
$$

and it follows from (8) and (9) that

$$
\Omega^{\prime}=2\left(\Sigma_{+}^{2}+R^{2} \cos 2 \psi\right) \Omega .
$$

For future reference, we introduce an auxiliary variable $Z$ according to

$$
Z=\frac{M R^{2}}{\sqrt{\Omega}}
$$

which satisfies

$$
Z^{\prime}=\left[-1-\left(2+\Sigma_{+}\right) \cos 2 \psi-3 R^{2} M \sin 2 \psi\right] Z,
$$

as follows from (9), (10), and (13).

\section{$3 \quad$ Limits at late times}

In this Section we find the limits of $\Sigma_{+}$and $R$ as $\tau \rightarrow \infty$. The limit of $M$ is

$$
\lim _{\tau \rightarrow \infty} M=0
$$

which follows immediately from (5). Since $\psi^{\prime}$ in (11) is proportional to $M^{-1}$, the trigonometric functions in (8)-(10) will oscillate increasingly rapidly as $\tau \rightarrow+\infty$. To facilitate the study of the behaviour at late times, we therefore introduce new gravitational variables, $\bar{\Sigma}_{+}, \bar{R}$, and $\bar{M}$, according to

$$
\begin{aligned}
\bar{\Sigma}_{+} & =\Sigma_{+}-\frac{1}{4} M\left(1+\Sigma_{+}\right) R^{2} \sin 2 \psi, \\
\bar{R} & =\frac{R}{1+\frac{1}{4} M\left(R^{2}-1-\Sigma_{+}\right) \sin 2 \psi}, \\
\bar{M} & =\frac{M}{1+\frac{1}{2} R^{2} \sin 2 \psi} .
\end{aligned}
$$

We likewise replace the auxiliary variables $Z$ and $\Omega$ by the variables $\bar{Z}$ and $\bar{\Omega}$, defined by

$$
\begin{aligned}
& \bar{Z}=\frac{Z}{1+\frac{1}{2} M\left(1+\Sigma_{+}\right) \sin 2 \psi}, \\
& \bar{\Omega}=\frac{\Omega}{1+\frac{1}{2} M R^{2} \sin 2 \psi} .
\end{aligned}
$$


These new variables are defined so as to ensure that the oscillatory terms in their evolution equations tend to zero as $\tau \rightarrow+\infty$. This follows since the oscillatory terms in (8)-(10) are bounded as $\tau \rightarrow+\infty$. The evolution equations, which follow from (8)-(11), (13), and (15), have the form

$$
\begin{aligned}
\bar{\Sigma}_{+}^{\prime} & =-\bar{R}^{2}-\bar{\Sigma}_{+}\left(1-\bar{\Sigma}_{+}\right)+M R^{2} B_{\bar{\Sigma}_{+}}, \\
\bar{R}^{\prime} & =\left[\bar{\Sigma}_{+}+\bar{\Sigma}_{+}^{2}+M B_{\bar{R}}\right] \bar{R}, \\
\bar{M}^{\prime} & =-\left[\left(1+\bar{\Sigma}_{+}\right)^{2}+M B_{\bar{M}}\right] \bar{M}, \\
\bar{Z}^{\prime} & =\left[-1+M B_{\bar{Z}}\right] \bar{Z}, \\
\bar{\Omega}^{\prime} & =2\left[\bar{\Sigma}_{+}^{2}+M R^{2} B_{\bar{\Omega}}\right] \bar{\Omega},
\end{aligned}
$$

where the $B$ 's are bounded functions as $\tau \rightarrow+\infty$. Equations 222)-(26) depend explicitly on $\psi$ through the B's which are all multiplied by a factor of $M$. This fact, in conjunction with (16), implies that the oscillatory terms in (22)-(26) tend to zero as $\tau \rightarrow+\infty$, as desired. The structure of (22)-(26) now allows the limits of $\bar{\Sigma}_{+}$and $R$ as $\tau \rightarrow+\infty$ to be found, and subsequently the corresponding limits of $\Sigma_{+}$and $R$. The main result is contained in the the following theorem.

\section{Theorem 1}

Any solution of the differential equations (8)-(11) with $\Omega>0$ that is not LRS, satisfies (16) and

$$
\lim _{\tau \rightarrow+\infty} \Sigma_{+}=0, \quad \lim _{\tau \rightarrow+\infty} R=0
$$

with

$$
\lim _{\tau \rightarrow+\infty} \frac{M}{R}=0 .
$$

Proof: The proof, which is based on equations (22)-(26), is given in Appendix A.

In the next Section we will consider the physical implications of this theorem as regards isotropization of the cosmological model.

\section{Anisotropy of the models at late times}

To quantify the deviation of non-tilted Bianchi $\mathrm{VII}_{0}$ models from the flat FL model, we introduce a set of dimensionless anisotropy scalars, $\{\Sigma, \mathcal{S}, \mathcal{W}\}$, all of which are zero for any FL model. The anisotropy in the expansion of a cosmological model is described by the shear scalar $\Sigma$, defined by

$$
\Sigma^{2}=\frac{\sigma_{a b} \sigma^{a b}}{6 H^{2}}
$$

where $\sigma_{a b}$ is the rate of shear tensor of the fluid congruence 3 . The anisotropy in the spatial geometry is described by the scalar $\mathcal{S}$, defined by

$$
\mathcal{S}^{2}=\frac{{ }^{3} S_{a b}{ }^{3} S^{a b}}{6 H^{2}}
$$

where ${ }^{3} S_{a b}$ is the trace-free spatial Ricci tensor (see Wainwright \& Ellis (1997), page 29). Finally, the intrinsic anisotropy of the gravitational field is described by the scalar $\mathcal{W}$, defined by

$$
\mathcal{W}^{2}=\frac{E_{a b} E^{a b}+H_{a b} H^{a b}}{6 H^{2}},
$$

where $E_{a b}$ and $H_{a b}$ are the electric and magnetic part of the Weyl tensor with respect to the fluid congruence respectively (see Wainwright \& Ellis (1997), page 19). The scalars $\mathcal{S}$ and $\mathcal{W}$ describe the dynamical

\footnotetext{
${ }^{3}$ It is this tensor that is parametrized by the variables $\sigma_{ \pm}$in (3).
} 
importance of the spatial curvature and the Weyl curvature compared to the overall expansion of the universe respectively. The use of expansion-normalized anisotropy scalars dates back to the important paper on cosmological observations by Kristian \& Sachs (1966) (see page 398), where they showed that various geometrical quantities, including the Weyl tensor can, in principle, be restricted by observations. For nontilted Bianchi models, a zero shear tensor, $\sigma_{a b}=0$, implies that the Weyl tensor is zero, and can thus be viewed as characterizing the FL models. Restricting the expansion-normalized shear scalar $\Sigma$ to be small, however, does not guarantee that the expansion-normalized Weyl tensor, as described by $\mathcal{W}$, is small. For a model to be close to an FL model as $\tau \rightarrow+\infty$, we therefore require that

$$
\Sigma \ll 1, \quad \mathcal{S} \ll 1, \quad \mathcal{W} \ll 1,
$$

as $\tau \rightarrow+\infty$. The limits of the anisotropy scalars $\{\Sigma, \mathcal{S}, \mathcal{W}\}$ as $\tau \rightarrow+\infty$ for non-tilted Bianchi VII $_{0}$ models with a radiation fluid are given by the following theorem.

\section{Theorem 2}

For any non-tilted radiation-filled Bianchi VII $I_{0}$ cosmological model that is not LRS, the density parameter satisfies

$$
\lim _{\tau \rightarrow+\infty} \Omega=1,
$$

and the anisotropy scalars satisfy

$$
\lim _{\tau \rightarrow+\infty} \Sigma=0, \quad \lim _{\tau \rightarrow+\infty} \mathcal{W}=+\infty
$$

and

$$
\limsup _{\tau \rightarrow+\infty} \mathcal{S}=+\infty, \quad \liminf _{\tau \rightarrow+\infty} \mathcal{S}=0 .
$$

Proof: This theorem is an immediate consequence of Theorem 1, once $\Sigma, \mathcal{S}$, and $\mathcal{W}$ are expressed in terms of the variables $\Sigma_{+}, R, M$, and $\psi$. In WHU it was shown that

$$
\Sigma^{2}=\Sigma_{+}^{2}+R^{2} \cos ^{2} \psi,
$$

and

$$
\mathcal{W}=\frac{2 R}{M}[1+\mathcal{O}(M)],
$$

(see WHU, equations (3.33) and (3.39)). Since ${ }^{3} S_{a b}$ is diagonal for Bianchi type $\mathrm{VII}_{0}$ models, it follows that

$$
\mathcal{S}^{2}=\mathcal{S}_{+}^{2}+\mathcal{S}_{-}^{2},
$$

(see Wainwright \& Ellis (1997), page 123), where

$$
\mathcal{S}_{+}=\frac{1}{2 H}\left({ }^{3} S_{22}+{ }^{3} S_{33}\right), \quad \mathcal{S}_{-}=\frac{\sqrt{3}}{2 H}\left({ }^{3} S_{22}-{ }^{3} S_{33}\right) .
$$

Specializing equation (6.36) in Wainwright \& Ellis (1997) to Bianchi $\mathrm{VII}_{0}$ models yields

$$
\mathcal{S}_{+}=2 N_{-} N_{+} \quad \mathcal{S}_{-}=2 N_{-}^{2},
$$

which, in conjunction with (7), implies that

$$
\mathcal{S}^{2}=\frac{4 R^{2} \sin ^{2} \psi}{M^{2}}\left[1+\mathcal{O}\left(M^{2}\right)\right]
$$

Equations (31), (32), and (36) and Theorem 1 now give Theorem 2.

The limits in Theorem 2 show that radiation filled Bianchi $\mathrm{VII}_{0}$ models isotropize with respect to the shear, but not with respect to the spatial curvature or the Weyl tensor. In view of (30), therefore, a general non-tilted Bianchi $\mathrm{VII}_{0}$ model with a radiation-fluid source is not close to any FL model at late times. 


\section{The asymptotic solution at late times}

In this Section we determine the asymptotic form of the gravitational field variables $\Sigma_{+}, R, M$, and $\psi$ as $\tau \rightarrow+\infty$. From these expressions, the asymptotic forms of the anisotropy scalars of the previous Section can subsequently be found. Theorem 1, in conjunction with (24), implies that

$$
M=\mathcal{O}\left(\mathrm{e}^{-b \tau}\right)
$$

as $\tau \rightarrow+\infty$, where the constant $b$ can be written as $b=1-\delta$. Here the constant $\delta>0$ can be chosen arbitrarily small. It now follows from (25) that there exists a constant $C_{Z}$ such that $\bar{Z}=C_{Z} \mathrm{e}^{-\tau}\left[1+\mathcal{O}\left(\mathrm{e}^{-b \tau}\right)\right]$. Hence, using (20), we find

$$
Z=C_{Z} \mathrm{e}^{-\tau}\left[1+\mathcal{O}\left(\mathrm{e}^{-b t}\right)\right]
$$

as $\tau \rightarrow+\infty$. The asymptotic behaviour of $\bar{\Sigma}_{+}$and $\bar{R}$, and hence of $\Sigma_{+}$and $R$, is governed by (22) and (23). We begin our analysis by considering the differential equations in $\mathbb{R}^{2}$ obtained by omitting the terms in (22) and (23) that involve $M$. The resulting differential equations are

$$
\begin{aligned}
\hat{R}^{\prime} & =\hat{\Sigma}_{+}\left(1+\hat{\Sigma}_{+}\right) \hat{R}, \\
\hat{\Sigma}_{+}^{\prime} & =-\hat{\Sigma}_{+}\left(1-\hat{\Sigma}_{+}^{2}\right)-\hat{R}^{2},
\end{aligned}
$$

which we will refer to as the truncated differential equations. Their solutions will be denoted by $\hat{\Sigma}_{+}$and $\hat{R}$. Since the proof in Appendix A that any solution of (22) and (23) with $\bar{R}>0$ and $\bar{\Omega}>0$ satisfies $\lim _{\tau \rightarrow+\infty} \bar{\Sigma}_{+}=0=\lim _{\tau \rightarrow+\infty} \bar{R}$ also applies to the truncated differential equtions (39) and (40), it follows that

$$
\lim _{\tau \rightarrow+\infty} \hat{\Sigma}_{+}=0, \quad \lim _{\tau \rightarrow+\infty} \hat{R}=0,
$$

for any solution with $\hat{R}>0$ and $\hat{\Omega}=1-\hat{\Sigma}_{+}^{2}-\hat{R}^{2}>0$. In other words, the origin is the future attractor for the truncated equations (39) and (40). A straightforward calculation shows that this equilibrium point is non-hyperbolic, with eigenvalues $\lambda_{\hat{\Sigma}_{+}}=-1$ and $\lambda_{\hat{R}}=0$. It will therefore be necessary to consider centre

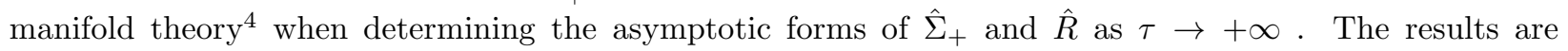
summarized in the following theorem.

\section{Theorem 3}

For any solution of the truncated differential equations (39) and (40) with $\hat{\Omega}>0$, and $\hat{R}>0$, there exists a constant $C_{R}$ such that

$$
\begin{gathered}
\hat{R}=\frac{1}{\sqrt{2 \tau}}\left[1-\frac{1}{4 \tau}\left(\ln \tau+C_{R}\right)+\mathcal{O}\left(T^{2}\right)\right], \\
\hat{\Sigma}_{+}=-\frac{1}{2 \tau}\left[1-\frac{1}{2 \tau}\left(\ln \tau+C_{R}-2\right)+\mathcal{O}\left(T^{2}\right)\right],
\end{gathered}
$$

where $T=\frac{\ln \tau}{\tau}$.

Proof: The proof is given in Appendix B.

The next step is to relate the solutions of (22) and (23) to the asymptotic forms in Theorem 3. An important consequence of Theorem 3 is that $\hat{\Sigma}_{+}$and $R$ exhibit a power-law decay to zero. This is the typical behaviour for non-hyperbolic equilibrium points. Since (22) and (23) differ from the truncated differential equations (39) and (40) by the addition of exponentially decaying terms, it is plausible that the asymptotic decay rates of the solutions will not be affected. We therefore conjecture that any solution $\left(\bar{R}, \bar{\Sigma}_{+}\right)$of (22) and (23) with

\footnotetext{
${ }^{4}$ For an introduction to centre manifold theory, see, for example, Carr (1981).
} 
$\bar{R}>0$ and $\bar{\Omega}>0$ will have the same asymptotic form as the solutions $\left(\hat{R}, \hat{\Sigma}_{+}\right)$of the truncated differential equations 39 and (40) as given in Theorem 3, i.e.,

$$
\begin{aligned}
\bar{R} & =\frac{1}{\sqrt{2 \tau}}\left[1-\frac{1}{4 \tau}\left(\ln \tau+C_{R}\right)+\mathcal{O}\left(T^{2}\right)\right], \\
\bar{\Sigma}_{+} & =-\frac{1}{2 \tau}\left[1-\frac{1}{2 \tau}\left(\ln \tau+C_{R}-2\right)+\mathcal{O}\left(T^{2}\right)\right] .
\end{aligned}
$$

The dependence of the constant $C_{R}$ on the initial conditions will be different than that of the solution (42)(43). We have not been able to give a proof of the above conjecture in general, but in Appendix $\mathrm{C}$ we give a proof of a related but simpler result. Assuming (44) and (45), it follows from (17), (18), and (37) that

$$
\begin{aligned}
R & =\frac{1}{\sqrt{2 \tau}}\left[1-\frac{1}{4 \tau}\left(\ln \tau+C_{R}\right)+\mathcal{O}\left(T^{2}\right)\right], \\
\Sigma_{+} & =-\frac{1}{2 \tau}\left[1-\frac{1}{2 \tau}\left(\ln \tau+C_{R}-2\right)+\mathcal{O}\left(T^{2}\right)\right] .
\end{aligned}
$$

The asymptotic form of $M$ can be obtained from (46) and (47) using (14) and (38). The result is

$$
M=C_{M} \tau \mathrm{e}^{-\tau}\left[1+\frac{1}{4 \tau}\left(2 \ln \tau+2 C_{R}-1\right)+\mathcal{O}\left(T^{2}\right)\right]
$$

where $C_{M}=2 C_{Z}$. Subsequently, by using (11), we find

$$
\psi^{\prime}=\frac{2 \mathrm{e}^{\tau}}{C_{M} \tau}\left[1-\frac{1}{4 \tau}\left(2 \ln \tau+2 C_{R}-1\right)+\mathcal{O}\left(T^{2}\right)\right] .
$$

This equation determines $\psi$ up to an additive constant $C_{\psi}$, although $\psi$ cannot be expressed in elementary terms.

The Hubble variable $H$ can be determined algebraically through the relation $\Omega=\frac{\mu}{3 H^{2}}$, since, for a radiation fluid, the quantity $\mu l^{4}$ is a constant. Here $l$ is the length scale function, which is related to $\tau$ according to

$$
l=C_{l} \mathrm{e}^{\tau},
$$

where $C_{l}$ is a constant (see WHU, equation (1.3)). It follows, using (4), (46), (47), and (50) that $H$ is given by

$$
H=C_{H} \mathrm{e}^{-2 \tau}\left[1+\frac{1}{4 \tau}+\mathcal{O}\left(\frac{\ln \tau}{\tau^{2}}\right)\right]
$$

where $C_{H}$ is a constant. Finally, by using (51), in conjunction with (6), the relationship between the cosmological clock time $t$ and the Hubble time $\tau$ can be found. By choosing appropriate initial conditions for $t$, we obtain

$$
t=\frac{1}{2 C_{H}} \mathrm{e}^{2 \tau}\left[1-\frac{1}{4 \tau}+\mathcal{O}\left(\frac{\ln \tau}{\tau^{2}}\right)\right],
$$

as $\tau \rightarrow+\infty$. The freedom to shift the time-variable according to $\tau \rightarrow \tau+C$, where $C$ is a constant, can be used to fix one of the constants, say $C_{l}=1$. There thus remain four essential constants of integration, namely $C_{R}, C_{\psi}, C_{M}$, and $C_{H}$. This confirms that (46)-(52) describe the asymptotic form of the general Bianchi $\mathrm{VII}_{0}$ solution with a radiation fluid source.

We conclude this Section by giving the asymptotic form of the various scalars of physical interest in terms of the clock time $t$. The asymptotic form of the anisotropy scalars $\Sigma^{2}, \mathcal{W}^{2}$ and $\mathcal{S}^{2}$ follow immediately from (31), (32), (46)-(49) and (52). The asymptotic form of the Hubble variable $H$ and the radiation density $\mu$ 
are obtained from (2), (12), (46), (47), (51) and (52). For convenience we introduce new constants $A$ and $\alpha$ according to

$$
A=2 C_{H}, \quad \alpha=\frac{4}{C_{M}} .
$$

Note that $A$ has dimension of $(\text { time })^{-1}$, while $\alpha$ is dimensionless. The results are

$$
\begin{aligned}
H & \approx \frac{1}{2 t}\left(1+\frac{1}{2 \ln (A t)}\right), \\
\mu & \approx \frac{3}{4 t^{2}}\left(1-\frac{1}{\ln (A t)}\right), \\
\Sigma^{2} & \approx \frac{\cos ^{2} \psi}{\ln (A t)}+\frac{1}{[\ln (A t)]^{2}}, \\
\mathcal{W}^{2} & \approx \frac{\alpha(A t)}{[\ln (A t)]^{3}}, \\
\mathcal{S}^{2} & \approx \mathcal{W}^{2} \sin ^{2} \psi,
\end{aligned}
$$

where

$$
\frac{d \psi}{d t} \approx \frac{\alpha A \sqrt{A t}}{2 \ln (A t)}
$$

It is understood that these asymptotic forms are valid for values of $A$ and $t$ such that $\ln (A t) \gg 1$. As mentioned in Section 1, these asymptotic forms could describe the dynamics of the universe in the final stages of the radiation-dominated epoch, which ends at a time $t=t_{\text {eq }}$, when the matter density first equals the radiation density. In order to describe this situation, the constant $A$ must satisfy

$$
\ln \left(A t_{\mathrm{eq}}\right) \gg 1 .
$$

It follows from (56), in conjunction with (59), that the constant $\alpha$ determines how rapidly $\Sigma$ oscillates. It also determines the magnitude of the Weyl scalar $\mathcal{W}$ at $t=t_{\text {eq }}$ (see (57)). We shall discuss the significance of the above results in Section 7.

\section{Relation with the metric approach}

The late time behaviour of Bianchi $\mathrm{VII}_{0}$ models with a radiation-fluid source has been considered by Doroshkevich et al (1973) using metric variables. In our approach, the asymptotic behaviour of the metric components can be found directly from the behaviour of the orthonormal frame variables in (46)-(51), and we now do this for the purpose of comparison.

For a Bianchi type $\mathrm{VII}_{0}$ model, a set of group-invariant and time-independent 1 -forms, $\boldsymbol{\omega}^{\alpha}, \alpha=1,2,3$ satisfying

$$
d \boldsymbol{\omega}^{1}=0, \quad d \boldsymbol{\omega}^{2}=\boldsymbol{\omega}^{3} \wedge \boldsymbol{\omega}^{1}, \quad d \boldsymbol{\omega}^{3}=\boldsymbol{\omega}^{1} \wedge \boldsymbol{\omega}^{2},
$$

can be introduced for which the line element can be written

$$
d s^{2}=-d t^{2}+g_{\alpha \beta} \boldsymbol{\omega}^{\alpha} \boldsymbol{\omega}^{\beta} .
$$

Here $t$ is (as previously) the cosmological clock time. For a non-tilted Bianchi VII ${ }_{0}$ model, $g_{\alpha \beta}$ can be diagonalized. Using the variables of Misner (1969) to parametrize $g_{\alpha \beta}$, the line element 62 can be written as

$$
d s^{2}=-d t^{2}+l^{2}\left[\mathrm{e}^{-4 \beta^{+}}\left(\boldsymbol{\omega}^{1}\right)^{2}+\mathrm{e}^{2\left(\beta^{+}+\sqrt{3} \beta^{-}\right)}\left(\boldsymbol{\omega}^{2}\right)^{2}+\mathrm{e}^{2\left(\beta^{+}-\sqrt{3} \beta^{-}\right)}\left(\boldsymbol{\omega}^{3}\right)^{2}\right] .
$$


The relationships between the metric variables $\beta^{ \pm}$and the orthonormal frame variables for Bianchi class A models are given in chapter 9 of Wainwright \& Ellis (1997). Specializing these relationships to Bianchi VII ${ }_{0}$ models yields

$$
N_{+}+\sqrt{3} N_{-}=\frac{1}{H l} \mathrm{e}^{2\left(\beta^{+}+\sqrt{3} \beta^{-}\right)}, \quad N_{+}-\sqrt{3} N_{-}=\frac{1}{H l} \mathrm{e}^{2\left(\beta^{+}-\sqrt{3} \beta^{-}\right)} .
$$

Isolating $\beta^{+}$and $\beta^{-}$leads to the following expressions

$$
\tanh \left(2 \sqrt{3} \beta^{-}\right)=\sqrt{3} R M \sin \psi, \quad \mathrm{e}^{4 \beta^{+}}=\frac{l^{2} H^{2}}{M^{2}}\left(1-3 R^{2} M^{2} \sin ^{2} \psi\right),
$$

(see Appendix C in WHU). Using (46), (47), 48), and (49), it follows that

$$
\begin{aligned}
& \beta^{+} \approx-\frac{1}{2} \ln \tau-\frac{1}{4 \tau} \ln \tau-\frac{1}{4 \tau}\left(C_{R}-1\right), \\
& \beta^{-} \approx \frac{C_{H} C_{l}}{2 \sqrt{2}} \mathrm{e}^{-\tau} \sqrt{\tau}\left[1+\frac{1}{4 \tau} \ln \tau+\frac{1}{4 \tau}\left(C_{R}-1\right)\right] \sin \psi .
\end{aligned}
$$

To facilitate a comparison with the asymptotic expressions of Doroshkevich et al (1973), we note that their variables $\gamma, \mu$ and $\left(\lambda_{1} \lambda_{2}\right)^{1 / 2}$ are related to the variables used in this paper by

$$
\gamma=l^{6}, \quad \mu=4 \sqrt{3} \beta^{-}, \quad\left(\lambda_{1} \lambda_{2}\right)^{1 / 2}=l^{2} \mathrm{e}^{2 \beta^{+}},
$$

and that their time variable $T$ (the so-called BKL time) is related to $\tau$ by

$$
\frac{d \tau}{d T}=H l^{3} \quad \Rightarrow \quad T \approx-\left(C_{H} C_{l}\right)^{-1} \mathrm{e}^{-\tau}\left(1-\frac{1}{4 \tau}\right) .
$$

We note that $T$ approaches zero from below as $\tau \rightarrow+\infty$. Doroshkevich et al (1973) give the following asymptotic forms:

$$
l^{2} \mathrm{e}^{2 \beta^{+}} \approx D_{1} \frac{\theta(T)}{T^{2}}, \quad \beta^{-} \approx D_{2} \theta(T)^{-1 / 2} T \sin \psi(T), \quad \frac{d \psi}{d T} \approx \frac{D_{3} \theta(T)}{T^{2}},
$$

see Appendix I, equation (I.14). Note that we have relabeled their constants. The function $\theta(T)$ in Doroshkevich et al (1973) is given by

$$
\theta(T) \approx \frac{1}{\nu}\left[1-\frac{D_{4}}{\nu}-\frac{\ln \nu}{2 \nu}\right]
$$

where $\nu=\ln \left(D_{5} / T\right)$ (see Appendix I, equation (I.15)). Using (69) in (71) yields

$$
\theta \approx \frac{1}{\tau}\left(1-\frac{D_{*}}{\tau}-\frac{\ln \tau}{2 \tau}\right)
$$

where the constant $D_{*}$ is a combination of the constants $C_{H}, C_{l}, D_{4}$ and $D_{5}$. Using (69) and (72) in (70) yields (66) and (67) if we identify $C_{R}=2 D_{*}, D_{1}=C_{H}^{-2} C_{l}^{-4}$, and $D_{2}=-\sqrt{6} / D_{1}$. From (69) it also follows that the evolution equation for $\psi$ in (70) is (asymptotically) equivalent to (49) if we identify $D_{3}=2 / D_{1}$.

\section{Discussion}

In this paper we have given a complete analysis of the behaviour at late times of non-tilted Bianchi VII $_{0}$ cosmologies filled with radiation. In particular, we have established rigorously the late time behaviour of the dimensionless anisotropy scalars $\Sigma, \mathcal{W}$, and $\mathcal{S}$, that determine the extent to which the models deviate from an FL model (Theorem 2). In addition, we have derived the asymptotic form as $\tau \rightarrow+\infty$ of the general solution, although in this regard we have had to rely on a heuristic argument at one stage of the analysis.

Our results show that the Bianchi $\mathrm{VII}_{0}$ radiation models differ from the dust models in two significant ways: 
1. for dust models, the dimensionless Weyl scalar $\mathcal{W}$ has a finite non-zero limit (see Theorem 2.4 in WHU), while for radiation-filled models, $\mathcal{W}$ increases without bound,

2. for dust models, the dimensionless shear scalar $\Sigma$ tends to zero at an exponential rate in terms of the Hubble time $\tau$, while for radiation models $\Sigma$ decays at a power law rate in terms of $\tau$.

The results also shed new light on the dynamics of the radiation models at late times. These models isotropize as regards the shear (i.e. $\Sigma \rightarrow 0$ ), but not as regards the Weyl curvature and the anisotropic spatial curvature (i.e. $\mathcal{W} \nrightarrow 0, \mathcal{S} \nrightarrow 0$ ). Equations (54)-(58) give the asymptotic dependence of the various scalars of physical interest on the clock time $t$. We note that the leading order terms of $H$ and $\mu$ in (54) and (55) give the flat FL radiation solution, and that, in addition, the shear scalar $\Sigma$ in (56) tends to zero. The behaviour of $\mathcal{W}$ and $\mathcal{S}$ in (57) and (58), however, implies that the solution is not close to the flat FL model at late times. Moreover, since $\mathcal{W}$ diverges at late times, the models exhibit the phenomenon of Weyl curvature dominance, i.e., the Weyl curvature plays a dominant role in determining the dynamics. These considerations show that solving the linearized Einstein field equations can lead to incorrect conclusions regarding the dynamics of the full equations. It was pointed out in WHU (see pages 2588-89) that some aspects of the late time evolution of dust models of Bianchi $\mathrm{VII}_{0}$ are correctly described by linearizing the Bianchi $\mathrm{VII}_{0}$ evolution equations about the flat $\mathrm{FL}$ model. On the other hand, for radiation models, the linearized solutions totally fail to describe the asymptotic behaviour of the shear, the Weyl curvature, and the anisotropic spatial curvature at late times 5 , implying that this behaviour is a fully non-linear phenomenon.

The rate at which the shear scalar $\Sigma$ tends to zero requires further comment. Equation (56) shows that the asymptotic form of $\Sigma^{2}$ is the sum of two terms. One of them is oscillatory and decays as $(\ln A t)^{-1}$, while the other is non-oscillatory and decays as $(\ln A t)^{-2}$. It appears that the dominant oscillatory term has been inadvertently omitted in the literature giving the incorrect result that $\Sigma \approx \frac{1}{\ln (A t)}$ as $t \rightarrow \infty$. For example, Doroshkevich et al (1973) state that "the anisotropy of the deformation tensor, ..., decreases very slowly, proportionally to $(\ln t)^{-1}$ " (see page 741 ), but do not specify what normalization was used to calculate the "anisotropy of the deformation tensor". In a subsequent article, however, Novikov (1974), states the conclusion more explicitly, namely that the components of the shear tensor, when normalized with the Hubble scalar $H$, decay as $\left[\ln \left(t / t_{F}\right)\right]^{-1}$, (see equation (2) on page 275 , and the definition 5 of $\Delta H_{i} / H$ on page 274). This incorrect result was subsequently quoted by Barrow (1976) (see the equation for $\sigma / \theta$ in the case $p=\frac{1}{3} \mu$, on page 364 ).

As mentioned in Section 1, the late time behaviour if Bianchi $\mathrm{VII}_{0}$ radiation models could describe the dynamics of the universe in the final stages of the radiation dominated epoch. Our results show that on transition from the radiation-dominated epoch to the matter-dominated epoch at $t=t_{\text {eq }}$, the shear scalar would be small but the Weyl curvature scalar $\mathcal{W}$ would be large. These values of $\Sigma$ and $\mathcal{W}$ would then act as initial values, so to speak, for the matter-dominated epoch. Since $\Sigma \rightarrow 0$ and $\mathcal{W} \rightarrow \mathcal{W}_{\infty}$, where $\mathcal{W}_{\infty}$ is a positive constant, as $t \rightarrow \infty$ for a dust model, the shear scalar would remain small, but the dynamical significance of $\mathcal{W}$ would persist. It would be of interest to investigate density perturbations in such a model, since they could well behave differently than in a perturbed FL model.

\section{Acknowledgements}

The authors wish to thank David Siegel for supplying the proof of Proposition 1 in Appendix C. The research was supported in part by a grant from the Natural Sciences \& Engineering Research Council of Canada (JW), Gålöstiftelsen (USN), Svenska Institutet (USN), Stiftelsen Blanceflor (USN) and the University of Waterloo (USN, MH).

\footnotetext{
${ }^{5}$ Compare equation (4.3) in WHU, setting $\beta=0$ (i.e. $\gamma=\frac{4}{3}$ ), with our equations (46)-(48).

${ }^{6}$ We note that our shear variables $\Sigma_{ \pm}$are linear combinations of the $\Delta H_{i} / H$ in Novikov (1974).
} 


\section{Appendix A: Proof of Theorem 1}

In this Appendix we give the proof of Theorem 1 . The proof is based on the evolution equation for $\bar{\Omega}$ and the fact that $\bar{\Omega}$ is bounded. We begin by integrating equation (26) to obtain

$$
\frac{1}{2} \ln \frac{\bar{\Omega}}{\bar{\Omega}_{0}}=\int_{\tau_{0}}^{\tau} \bar{\Sigma}_{+}^{2} d s+\int_{\tau_{0}}^{\tau} M \bar{R}^{2} B_{\bar{\Omega}} d s,
$$

where $\bar{\Omega}_{0}=\bar{\Omega}\left(\tau_{0}\right)$ and the initial time $\tau_{0}$ is chosen such that $M \leq 1$ for all $\tau \geq \tau_{0}$. Equation (25) implies that

$$
\bar{Z}=\mathcal{O}\left(\mathrm{e}^{(-1+\delta) \tau}\right)
$$

where $\delta$ can be chosen arbitrarily small 7 . We now require the fact that $\bar{\Omega}$ is bounded, which follows from (12), (16), and (21). Recalling the definitions (14) and (20), equation (74) implies

$$
\bar{M} \bar{R}^{2}=\mathcal{O}\left(\mathrm{e}^{(-1+\delta) \tau}\right)
$$

and hence

$$
\int_{\tau_{0}}^{\tau} M \bar{R}^{2} B_{\bar{\Omega}} d s=C+\mathcal{O}\left(\mathrm{e}^{(-1+\delta) \tau}\right)
$$

where $C$ is a constant depending on the initial conditions at $\tau=\tau_{0}$. Using (76) and the fact that $\Omega$ is positive and bounded, we conclude from (73) that $I(\tau)=\int_{\tau_{0}}^{\tau} \bar{\Sigma}_{+}^{2} d s$ is bounded above for all $\tau \geq \tau_{0}$. Since the integrand is non-negative, the function $I(\tau)$ is monotonically decreasing, which implies that $\lim _{\tau \rightarrow+\infty} I(\tau)$ exists and is finite. It follows from (73), (76) and (21) that $\lim _{\tau \rightarrow+\infty} \bar{\Omega}$, and hence $\lim _{\tau \rightarrow+\infty} \Omega$ exists, i.e. there is a constant $L$, satisfying $0 \leq L \leq 1$, such that

$$
\lim _{\tau \rightarrow \infty} \Omega=L .
$$

In order to proceed, we need the following standard result

\section{Lemma 1}

Let $f(\tau)$ be a non-negative real-valued and Lipschitz continuous function on the interval $\tau_{0} \leq \tau<+\infty$. If

$$
\int_{\tau_{0}}^{\infty} f(\tau) d \tau
$$

is finite then $\lim _{\tau \rightarrow+\infty} f(\tau)=0$.

Since $\bar{\Sigma}_{+}$and $\bar{R}$ are bounded, it follows from (22) that $\left(\bar{\Sigma}_{+}^{2}\right)^{\prime}=2 \bar{\Sigma}_{+} \bar{\Sigma}_{+}^{\prime}$ is uniformly bounded for $\tau \geq \tau_{0}$. This implies that $\bar{\Sigma}_{+}$is Lipschitz continuous on the interval $\tau \geq \tau_{0}$ (see ???). We may now apply Lemma 1, with $f=\bar{\Sigma}_{+}^{2}$, to conclude that

$$
\lim _{\tau \rightarrow+\infty} \bar{\Sigma}_{+}=0
$$

and hence, from (16) and (17)

$$
\lim _{\tau \rightarrow+\infty} \Sigma_{+}=0
$$

From (幽, (18), (77), and (79), it follows that

$$
\lim _{\tau \rightarrow+\infty} R^{2}=\lim _{\tau \rightarrow+\infty} \bar{R}^{2}=1-L .
$$

\footnotetext{
${ }^{7}$ Since $M$ tends to zero and $B_{\bar{Z}}$ is bounded, one can choose $\tau_{0}$ such that $\left|M B_{1}\right|<\delta$ for $\tau>\tau_{0}$.
} 
Equation (22), in conjunction with (79), (80), and (16), now implies that $\lim _{\tau \rightarrow+\infty} \bar{\Sigma}_{+}^{\prime}$ exists, and that

$$
\lim _{\tau \rightarrow+\infty} \bar{\Sigma}_{+}^{\prime}=L-1 \text {. }
$$

This result contradicts (78) unless $L=1$. Using (80) we obtain

$$
\lim _{\tau \rightarrow+\infty} R=0 \text {. }
$$

To find the limit of the quotient $\frac{M}{R}$, we note that (23) and (24) can be combined to give

$$
\left(\frac{\bar{M}}{\bar{R}}\right)^{\prime}=\left(-1-3 \bar{\Sigma}_{+}-2 \bar{\Sigma}_{+}^{2}+M B_{*}\right)\left(\frac{\bar{M}}{\bar{R}}\right),
$$

where $B_{*}$ is a bounded function as $\tau \rightarrow+\infty$. It now follows from (78) and (16) that

$$
\lim _{\tau \rightarrow+\infty} \frac{\bar{M}}{\bar{R}}=0
$$

and hence that

$$
\lim _{\tau \rightarrow+\infty} \frac{M}{R}=0 .
$$

\section{Appendix B: Proof of theorem 3}

In this Appendix we give the proof of Theorem 3, using centre manifold theory. In developing this theory, Carr (1981) (see his equation (2.3.1)), considers a system of differential equations of the form

$$
\begin{aligned}
& x^{\prime}=A x+f(x, y), \\
& y^{\prime}=B y+g(x, y),
\end{aligned}
$$

where $x \in \mathbb{R}^{n}, y \in \mathbb{R}^{m}, A$ is a constant $n \times n$ matrix, $B$ is a constant $m \times m$ matrix, and $f: \mathbb{R}^{n} \times \mathbb{R}^{m} \rightarrow \mathbb{R}^{n}$ and $g: \mathbb{R}^{n} \times \mathbb{R}^{m} \rightarrow \mathbb{R}^{m}$ are $C^{2}$ functions that are zero and have zero derivatives at the equilibrium point $(x, y)=(0,0)$. It is assumed that the eigenvalues of $A$ have zero real parts and that the eigenvalues of $B$ have negative real parts. The assumption on $A$ means that the equilibrium point $(x, y)=(0,0)$ is non-hyperbolic, and hence that the linearization of (86) and (87) at this equilibrium point does not describe the behaviour of solutions near the equilibrium point.

By theorem 1 in Carr (1981), the differential equations (86)-87) has a local center manifold $y=h(x)$, where $h \in C^{2}$, and the flow on this center manifold is governed by the reduced differential equation

$$
u^{\prime}=f(u, h(u)),
$$

(see equation (2.4.1) in Carr (1981)). By theorem 2 in Carr (1981) if the equilibrium point $u=0$ of (88) is asymptotically stable then the equilibrium point $(x, y)=(0,0)$ of $(86)$ - 87 ) is also asymptotically stable. In addition, if $(x(t), y(t))$ is a solution of (86)-(87) with $(x(0), y(0))$ sufficiently close to the origin, then there exists a solution $u(t)$ of 88$)$ such that

$$
\begin{aligned}
& x(t)=u(t)+\mathcal{O}\left(\mathrm{e}^{-\gamma t}\right), \\
& y(t)=h(u(t))+\mathcal{O}\left(\mathrm{e}^{-\gamma t}\right),
\end{aligned}
$$

\footnotetext{
${ }^{8}$ It is possible that the solution $u(t)$ is the zero solution, in which case $x(t)$ and $y(t)$ decay exponentially to zero. This special case will occur only if the initial point $(x(0), y(0))$ lies on the stable manifold of the equilibrium point $(x, y)=(0,0)$ of the differential equations (86)-87).
} 
where $\gamma>0$ is a constant depending only on the matrix $B$ (see Carr (1981), equation (2.4.5)). The implication of this result is that if the equilibrium point $u=0$ of (88) is asymptotically stable, one can determine the asymptotic behaviour of the solutions of (86)-(87) by finding the asymptotic behaviour of solutions of (88). In order to perform this analysis one has to approximate the center manifold $y=h(x)$, as follows. For a function $\phi: \mathbb{R}^{n} \rightarrow \mathbb{R}^{m}$ that is $C^{1}$ near the origin, define an operator $M$ by

$$
(M \phi)(x)=\phi^{\prime}(x)[A x+f(x, \phi(x))]-[B \phi(x)+g(x, \phi(x))] .
$$

By theorem 3 in Carr (1981), if $\phi(0)=0, \phi^{\prime}(0)=0$ and $(M \phi)(x)=\mathcal{O}\left(|x|^{q}\right)$ as $x \rightarrow 0$, where $q>1$, then

$$
|h(x)-\phi(x)|=\mathcal{O}\left(|x|^{q}\right),
$$

as $x \rightarrow 0$. In other words, the function $\phi(x)$ approximates the center manifold $y=h(x)$ with an accuracy determined by $q$.

The differential equation (39)-(40) is of the form (86)-(87) with $n=1, m=1$,

$$
\begin{gathered}
(x, y)=\left(\hat{R}, \hat{\Sigma}_{+}\right), \\
A=0, \quad B=1, \\
f(x, y)=x y(1+y), \quad g(x, y)=-x^{2}+y^{3} .
\end{gathered}
$$

In addition, we have shown that the equilibrium point $\left(\hat{R}, \hat{\Sigma}_{+}\right)$is asymptotically stable, so we can apply the above theory. Using (91) and (92), in conjunction with (93)-(95), we find that the center manifold is approximated by

$$
\hat{\Sigma}_{+}=-\hat{R}^{2}-2 \hat{R}^{4}+\mathcal{O}\left(\hat{R}^{6}\right)
$$

Substituting this in (39) gives the reduced differential equation (88) on the center manifold in the form

$$
\hat{R}^{\prime}=-\hat{R}^{3}-\hat{R}^{5}+\mathcal{O}\left(\hat{R}^{7}\right) .
$$

This differential equation is precisely equation (3.1.9) with $a=-2$, considered by Carr (1981), who shows that the asymptotic form of the solution is

$$
\hat{R}=\frac{1}{\sqrt{2 \tau}}\left[1-\frac{1}{4 \tau}\left(\ln \tau+C_{R}\right)+o\left(\tau^{-1}\right)\right],
$$

as $\tau \rightarrow+\infty$, where $C_{R}$ is a constant that depends on the initial conditions. The error bound $o\left(\tau^{-1}\right)$ can be

improved to $\mathcal{O}\left(\left(\frac{\ln \tau}{\tau}\right)^{2}\right)$, as in Theorem 3, by increasing the accuracy of the center manifold approximation (96) to

$$
\hat{\Sigma}_{+}=-\hat{R}^{2}-2 \hat{R}^{4}-11 \hat{R}^{6}+\mathcal{O}\left(\hat{R}^{8}\right)
$$

Finally, substituting (98) in (96) gives the asymptotic form of $\hat{\Sigma}_{+}$, as stated in Theorem 3.

\section{Appendix C}

In this Appendix we prove that any solution to the perturbed differential equation

$$
y^{\prime}=-y^{3}+\epsilon \mathrm{e}^{-\gamma \tau},
$$


where $\epsilon>0$ and $\gamma>0$ are constants, has the same asymptotic form as $\tau \rightarrow+\infty$ as the solutions to the unperturbed differential equation

$$
Y^{\prime}=-Y^{3}
$$

Equation (101) is a simplified form of the differential equation (97) that describes the flow on the center manifold in Appendix B, and the term $\epsilon \mathrm{e}^{-\gamma \tau}$ mimics the perturbation introduced by the exponentially decaying function $M$.

Proposition 1

For any solution $y(\tau)$ of $(100)$ there exists a solution $Y(\tau)$ of $(101)$ such that

$$
|y(\tau)-Y(\tau)|=\mathcal{O}\left(\mathrm{e}^{-\gamma \tau}\right)
$$

as $\tau \rightarrow+\infty$.

Proof: Let $y(\tau)$ be the solution of $(100)$ that satisfies the initial condition $y(0)=y_{0}$ where $y_{0}>0$. The differential equation (100) then implies that $y(t)$ remains positive and is bounded above. This, in turn, implies that the solution exists for all $\tau \geq 0$.

We first need to derive an upper bound for $\frac{1}{y(\tau)}$. The differential equation (100) implies that

$$
-\frac{y^{\prime}}{y^{3}} \leq 1
$$

which, when integrated from 0 to $\tau$, gives

$$
\frac{1}{y(\tau)^{2}} \leq 2 \tau+\frac{1}{y_{0}^{2}}
$$

We now define

$$
w(\tau)=\frac{1}{2 y(\tau)^{2}}-\tau
$$

The differential equation (100) implies that

$$
w^{\prime}=\frac{\epsilon \mathrm{e}^{-\gamma \tau}}{y(\tau)^{3}}
$$

Integrating (105) from 0 to $\tau$ gives

$$
w(\tau)-w(0)=-\epsilon \int_{0}^{\tau} \frac{\mathrm{e}^{-\gamma s}}{y(s)^{3}} d s
$$

Since $\frac{1}{y(s)^{3}}$ is bounded (see 103$)$ ), it follows that $\lim _{\tau \rightarrow+\infty} w(\tau)$ exists and equals $c$, where

$$
c=w(0)-\epsilon \int_{0}^{\infty} \frac{\mathrm{e}^{-\gamma s}}{y(s)^{3}} d s .
$$

Subtracting (107) from (106) and using (103) yields

$$
w(\tau)-c=\epsilon \int_{\tau}^{\infty} \frac{\mathrm{e}^{-\gamma s}}{y(s)^{3}} d s \leq \epsilon \int_{\tau}^{\infty} \mathrm{e}^{-\gamma s}\left(2 s+\frac{1}{y_{0}^{2}}\right)^{3 / 2} d s .
$$

Thus,

$$
w(\tau)=c+\mathcal{O}\left(\tau^{3 / 2} \mathrm{e}^{-\gamma \tau}\right)
$$


as $\tau \rightarrow+\infty$. It now follows from (104) that

$$
y(\tau)=\frac{1}{\sqrt{2} \sqrt{\tau+c+\mathcal{O}\left(\tau^{3 / 2} \mathrm{e}^{-\gamma \tau}\right)}},
$$

which can be rearranged to give

$$
y(\tau)=\frac{1}{\sqrt{2} \sqrt{\tau+c}}+\mathcal{O}\left(\mathrm{e}^{-\gamma \tau}\right),
$$

as $\tau \rightarrow+\infty$. Since the first term is a solution of (101), the proof is complete.

\section{References}

Barrow J 1976 Light elements and the isotropy of the universe Mon. Not. R. astr. Soc. 175 359-370

Carr J 1981 Application of center manifold theory (Springer Verlag: New York)

Doroshkevich A G, Lukash V N and Novikov I D 1973 The isotropization of homogeneous cosmological models Sov. Phys.-JETP 37 739-746

Ellis G F R and MacCallum M A H 1969 A class of homogeneous cosmological models Commun. Math. Phys. 12 108-141

Kristian J and Sachs R K 1966 Observations in cosmology Astrophys. J. 143 379-399

Lukash V N 1974 Some pecularities in the evolution of homogeneous anisotropic cosmological models Sov. Astron. 18 164-169

Lukash V N 1975 Gravitational waves that conserve the homogeneity of space Sov. Phys.-JETP $40792-799$

Lukash V N 1976 Physical Interpretation of homogeneous cosmological models Nuovo Cimento B 35 268-292

Misner C W 1969 Quantum Cosmology I Phys. Rev.186 1319-1326

Novikov I D 1974 Isotropization of homogeneous cosmological models in "Confrontation of cosmological theories with observational data", IAU Symposium No. 63, edited by Longair M S (Reidel:Dordrecht)

Wainwright J, Hancock M J and Uggla C 1999 Asymptotic self-similarity breaking at late times in cosmology Class. Quantum Grav.16 2577-2598

Wainwright J and Ellis G F R 1997 Dynamical systems in cosmology (Cambridge University Press: Cambridge) 